\title{
Time to sputum culture conversion and treatment outcome of patients with multidrug-resistant tuberculosis: a prospective cohort study from urban China
}

\begin{abstract}
To the Editor:
Sputum culture plays an important role in monitoring treatment response in patients with multidrug-resistant tuberculosis (MDR-TB), and sputum culture conversion is a clinical tool used to predict therapeutic efficacy [1]. Monthly culture monitoring is essential for earlier detection of treatment failure in patients with MDR-TB. More sensitive signals of nonresponse would further avoid adverse outcomes [2].

Evidence exists suggesting that sputum culture conversion after 2 months of treatment may be an early predictor of treatment success in patients with MDR-TB $[3,4]$. Although sputum culture conversion has been used substantially in clinical settings, few prospective cohort studies have investigated its efficacy after different durations of MDR-TB treatment, and no studies have been performed in China or India [5, 6].
\end{abstract}

We evaluated whether 2-, 3-, 6- or 24-month culture conversion predicted treatment success in patients with MDR-TB. The study was conducted in four cities (Xuzhou, Lianyungang, Zhenjiang and Nantong) in Jiangsu province, China. Xuzhou and Lianyungang, both located in the north of Jiangsu province, are economically underdeveloped areas with a higher TB burden while Zhenjiang, situated in the south, is a relatively rich city with a lighter TB burden, and Nantong, located in the middle of Jiangsu, is a middle-income developed city with a moderate TB burden [7]. All patients with MDR-TB were enrolled consecutively between December 2011 and March 2014. Patients with MDR-TB were identified at the time of diagnosis by regional reference laboratories using traditional drug sensitivity tests (DST), as previously described [8]. Patients whose sputum culture was negative at baseline were excluded. Once confirmed, every patient signed an informed consent form, after which a questionnaire designed by local investigators was administered to gather important demographic and clinical information. Sputum culture was performed monthly.

World Health Organization guidelines were consulted when defining study covariates and outcomes. Patients with MDR-TB were participants with Mycobacterium tuberculosis isolates resistance to at least isoniazid and rifampicin. A positive sputum culture was defined as Mycobacterium tuberculosis colony growth and a negative sputum culture as no colony growth [9]. Sputum culture conversion was defined as two consecutive negative cultures, collected at least 30 days apart. Sputum culture reversion to positive was defined as one or more subsequent positive results after initial conversion. Sensitivity was defined as the proportion of patients with sputum culture conversion among those with treatment success. Specificity was defined as the proportion of patients with nonconversion of sputum culture among those with poor outcome. Sensitivity of culture conversion was used as a predictor of treatment success and specificity of lack of culture conversion as a predictor of failure/death. Final outcomes included success, failure and death. End-of-treatment success was defined as completion of treatment or cure. Adverse outcomes were treatment failure or death. Sputum smear grading was as follows: $1+(3-9$ acid-fast bacilli (AFB) in 100 fields), 2+ (1-9 AFB in 10 fields), 3+ (1-9 AFB in 1 field) and $4+(\geqslant 10$ AFB in 1 field). Smear grade was dichotomised $\leqslant 1$ (included smear-negative and $1+$ ) and $>1$ (included smear-positive $2+, 3+, 4+$ ).

@ERSpublications

The timing of testing for culture conversion has potential as a marker of MDR-TB treatment success http://ow.ly/borO308pPXg

Cite this article as: Lu P, Liu Q, Martinez L, et al. Time to sputum culture conversion and treatment outcome of patients with multidrug-resistant tuberculosis: a prospective cohort study from urban China. Eur Respir J 2017; 49: 1601558 [https://doi.org/10.1183/13993003.01558-2016]. 
Cox proportional hazards modelling was used to estimate hazard ratios, with sputum culture conversion as the end-point. Kaplan-Meier survival curves were plotted (not shown), and differences between groups were estimated by the log-rank test. Odds ratios and Chi-squared tests were used to determine associations between conversion and treatment outcome.

Of 160 patients with pulmonary MDR-TB enrolled, 139 had recorded treatment outcomes. Median age was 51 (interquartile range (IQR) 35.8-60.0) years; median weight was 60 (IQR 53.0-65.0) kg; 99 (71.4\%) were male; 65 (46.8\%) had smoked previously or were current smokers at the time of treatment, and 26 (18.7\%) drank alcohol. Of 139 patients with MDR-TB, 84 (60.4\%) patients had a successful treatment outcome and 55 (39.6\%) either experienced treatment failure or died. In all, 106 (76.3\%) patients had sputum culture conversion. Median time to culture conversion among those who converted was 159 (IQR 127-212) days. Overall, the median sputum culture conversion time of patients with successful treatment outcomes was 92 days (95\% CI 85-99 days), which was much shorter $(\mathrm{p}<0.001)$ than for the patients with treatment failure or death (174 days, 95\% CI 0-513). 2-month conversion was not statistically significantly associated with treatment success $(\mathrm{p}=0.087)$. However, patients who converted sputum culture at 3 $(\mathrm{p}=0.007), 6(\mathrm{p}<0.001)$ and 24 months $(\mathrm{p}<0.001)$ had increased odds of treatment success compared to nonconverters. The overall sensitivity of sputum culture conversion at 2, 3, 6 and 24 months was 33.3\%, $52.4 \%, 90.5 \%$ and $95.2 \%$, respectively. The specificity of these markers was $80.0 \%, 70.9 \%, 56.4 \%$ and $52.7 \%$, respectively (table 1 ).

Earlier detection of participant nonresponse to treatment may allow clinicians to adjust treatment protocols and, subsequently, avoid poor outcomes [10]. A recent study conducted by MiTNICK et al. [2] demonstrated that monthly sputum culture outperformed bimonthly collection of sputum smear and culture in detecting patients with MDR-TB that failed treatment. These findings highlight the specific value of performing a prospective cohort study to investigate the efficacy of sputum culture conversion after different durations of MDR-TB treatment.

Anti-TB pharmacological treatment is adjusted according to the culture conversion of patients, resulting in decreased rates of adverse effects by reducing the number of given injectable drugs [11]. Furthermore, prolonging conversion time may increase the chance that patients transmit TB to other individuals in their social network. Therefore, sputum culture conversion has not only clinical significance but also important public health relevance. In the current study, sputum culture conversion at 2 months was not statistically associated with treatment success, whereas patients with sputum culture conversion at 3, 6 and 24 months had a significantly higher likelihood of treatment success compared with those who did not convert. Data from 12 randomised trials of patients with drug-susceptible tuberculosis suggested that 3-month culture conversion was superior to 2-month conversion as a surrogate for poor treatment outcome, but neither were ideal [12]. Although 2-month culture conversion is generally considered an early predictor of treatment success in patients with MDR-TB, its low sensitivity in detecting long-term treatment success is a cause for concern. A recent study by Kurbatova et al. [9] suggested that the overall association between culture conversion and treatment success is substantially stronger at 6 months compared with 2 months due to higher sensitivity. Our study corroborates this finding and also shows substantially lower specificity when evaluating treatment success at 6-versus 2-month culture conversion (specificity, 56.4\% versus

\begin{tabular}{|c|c|c|c|c|c|c|c|c|}
\hline 2 months & & & & & $33.3(23.4-44.5)$ & $80.0(67.0-89.6)$ & $71.8(55.1-85.0)$ & $44.0(34.1-54.3)$ \\
\hline Did not convert & $44(80.0)$ & $56(66.7)$ & 1 (reference) & & & & & \\
\hline Converted & $11(20.0)$ & 28 (33.3) & $2.0(0.9-4.5)$ & 0.087 & & & & \\
\hline 3 months & & & & & $52.4(41.2-63.4)$ & $70.9(57.1-82.4)$ & $73.3(60.3-83.9)$ & $49.4(37.9-60.9)$ \\
\hline 6 months & & & & & 90.5 (82.1-95.8) & $56.4(42.3-69.7)$ & $76.0(66.4-84.0)$ & $79.5(63.5-90.7)$ \\
\hline Did not convert & $31(56.4)$ & $8(9.5)$ & 1 (reference) & & & & & \\
\hline Converted & $24(43.6)$ & 76 (90.5) & $12.3(5.0-30.3)$ & $<0.001$ & & & & \\
\hline 24 months & & & & & $95.2(88.3-98.7)$ & $52.7(38.8-66.3)$ & 75.5 (66.2-83.3) & $87.9(71.8-96.6)$ \\
\hline Did not convert & 29 (52.7) & $4(4.8)$ & 1 (reference) & & & & & \\
\hline Converted & 26 (47.3) & 80 (95.2) & $22.3(7.2-69.4)$ & $<0.001$ & & & & \\
\hline
\end{tabular}

Data are presented as $\mathrm{n}(\%)$, unless otherwise stated. ${ }^{\#}$ : sputum culture conversion was defined as two consecutive negative cultures after the first sputum sample, collected at least 30 days apart. PPV: positive predictive value; NPV: negative predictive value. 
80.0\%). Our results suggest an intricate balance between high and low sensitivity and specificity, depending on the time point at which sputum culture testing occurs.

The low sensitivity at 2- and 3-month culture conversion suggests that many patients would be misclassified as having failed treatment, thereby underestimating overall therapeutic efficacy. Furthermore, such misclassification would result in replacement of effective drugs and termination of a potentially successful drug regimen. In our study, sputum culture conversion at 2 months had a sensitivity of 33.3\%, indicating that $66.7 \%$ of patients would be misclassified. However, the specificity for sputum culture conversion at the end of 6 months of treatment was $56.4 \%$, indicating that $43.6 \%$ of patients would be misclassified as effectively treated. These results indicate that missed opportunities to replace a more effective drug regimen for patients failing treatment is common, and may contribute to high death rates among patients with drug-resistant tuberculosis. Surrogate end-points should take into account the balance between predicting treatment success and failure accurately during treatment of drug resistance [9]. In our study, time to sputum culture conversion at 6 months was significantly associated with end-of-treatment outcomes. The high combined sensitivity and specificity was reached for sputum culture conversion status assessed at 6 months of treatment. Given the average 50-60\% prevalence of treatment success reported in MDR-TB cohorts worldwide [13, 14], PPVs were relatively high: 75.5\% for 6-month sputum culture conversion. Sputum culture conversion at 6 months may be a crucial indication for trials conducted to determine a highly effective and inexpensive standardised treatment.

In summary, our study suggests that sputum conversion at 6 months had a much higher sensitivity, but a substantially lower specificity, to predict MDR-TB treatment success compared with sputum conversion at 2 and 3 months.

Peng $\mathrm{Lu}^{1,5}$, Qiao Liu ${ }^{2,5}$, Leonardo Martinez ${ }^{3,5}$, Haitao Yang ${ }^{1,4}$, Wei $\mathrm{Lu}^{2}$, Xiaoyan Ding ${ }^{2}$ and Limei Zhu ${ }^{2}$

${ }^{1}$ Dept of Epidemiology and Biostatistics, School of Public Health, Nanjing Medical University, Nanjing, PR China. ${ }^{2}$ Dept of Chronic Communicable Disease, Center for Disease Control and Prevention of Jiangsu Province, Nanjing, PR China. ${ }^{3}$ Dept of Epidemiology and Biostatistics, University of Georgia School of Public Health, Athens, GA, USA. ${ }^{4}$ Institute of Parasitic Disease of Jiangsu Province, Wuxi, PR China. ${ }^{5}$ These authors contributed equally to this work.

Correspondence: Limei Zhu, 172 Jiangsu Rd, Nanjing, China. E-mail: jsjkmck@163.com

Received: July 152016 | Accepted after revision: Dec 112016

Support statement: This study was supported by the National Nature Science Foundation of China (81302480). The sponsors had no role in the study design or data analysis. Funding information for this article has been deposited with the Open Funder Registry.

Conflict of interest: None declared.

Acknowledgements

Author contributions: P. Lu and Q. Liu conceived the study, analysed the data and drafted the manuscript; L. Zhu, H. Yang, W. Lu and X. Ding participated in the study design and implemented the field investigation; and L. Martinez participated in the study design and helped draft the manuscript. All authors contributed to the study and have read and approved the final manuscript.

\section{References}

1 Nagaraja C, Shashibhushan BL, Asif M, et al. Pattern of drug-resistance and treatment outcome in multidrug-resistant pulmonary tuberculosis. Indian J Chest Dis Allied Sci 2012; 54: 23-26.

2 Mitnick CD, White RA, Lu C, et al. Multidrug-resistant tuberculosis treatment failure detection depends on monitoring interval and microbiological method. Eur Respir J 2016; 48: 1160-1170.

3 Basit A, Ahmad N, Khan AH, et al. Predictors of two months culture conversion in multidrug-resistant tuberculosis: findings from a retrospective cohort study. PLoS One 2014; 9: e93206.

4 Rodriguez M, Monedero I, Caminero JA, et al. Successful management of multidrug-resistant tuberculosis under programme conditions in the Dominican Republic. Int J Tuberc Lung Dis 2013; 17: 520-525.

5 Zhao Y, Xu S, Wang L, et al. National survey of drug-resistant tuberculosis in China. N Engl J Med 2012; 366: 2161-2170.

6 Gandhi NR, Nunn P, Dheda K, et al. Multidrug-resistant and extensively drug-resistant tuberculosis: a threat to global control of tuberculosis. Lancet 2010; 375: 1830-1843.

7 Shao Y, Yang D, Xu W, et al. Epidemiology of anti-tuberculosis drug resistance in a chinese population: current situation and challenges ahead. BMC Public Health 2011; 11: 110.

8 Liu Q, Zhu L, Shao Y, et al. Rates and risk factors for drug resistance tuberculosis in Northeastern China. BMC Public Health 2013; 13: 1171.

9 Kurbatova EV, Cegielski JP, Lienhardt C, et al. Sputum culture conversion as a prognostic marker for end-of-treatment outcome in patients with multidrug-resistant tuberculosis: a secondary analysis of data from two observational cohort studies. Lancet Respir Med 2015; 3: 201-209.

10 Falzon D, Jaramillo E, Schünemann HJ, et al. WHO guidelines for the programmatic management of drug-resistant tuberculosis: 2011 update. Eur Respir J 2011; 38: 516-528.

11 Velayutham B, Nair D, Kannan T, et al. Factors associated with sputum culture conversion in multidrug-resistant pulmonary tuberculosis. Int J Tuberc Lung Dis 2016; 1671-1676. 
12 Phillips PP, Fielding K, Nunn AJ. An evaluation of culture results during treatment for tuberculosis as surrogate endpoints for treatment failure and relapse. PLoS One 2013; 8: e63840.

13 Orenstein EW, Basu S, Shah NS, et al. Treatment outcomes among patients with multidrug-resistant tuberculosis: systematic review and meta-analysis. Lancet Infect Dis 2009; 9: 153-161.

14 World Health Organization. Global tuberculosis report 2016. Geneva, World Health Organization, 2016.

Copyright @ERS 2017

This ERJ Open article is open access and distributed under the terms of the Creative Commons Attribution Non-Commercial Licence 4.0. 